\title{
CONE TYPE MAJORIZATION AND ITS STRONG LINEAR PRESERVERS*
}

\author{
G. SANKARA RAJU KOSURU ${ }^{\dagger}$ AND SUBHAJIT SAHA
}

\begin{abstract}
This study introduces a novel notion, cone type majorization and characterizes the same. Further, the structure of linear preservers and strong linear preservers of this cone type majorization have been studied.
\end{abstract}

Key words. Majorization, Cone type majorization, Linear preserver, Stochastic matrix.

AMS subject classifications. 15A86, 15A04, 15A57, $15 \mathrm{~B} 51$.

1. Introduction. Majorization in finite dimensional spaces has been extensively studied because of its applications in a wide variety of areas such as mathematics, physics, engineering, and economics. Particularly in mathematics, majorization has applications in matrix analysis, operator theory, frame theory, and inequalities involving convex functions $([3,5,10])$. For a detailed overview of this subject, we refer to the book by Marshall and Olkin [8].

For any two vectors $x, y \in \mathbb{R}^{n}, x$ is said to be majorized by $y$, denoted by $x \prec y$, if

$$
\sum_{j=1}^{k} x_{j}^{\downarrow} \leq \sum_{j=1}^{k} y_{j}^{\downarrow} \quad(\text { for } k=1,2, \ldots, n-1) \quad \text { and } \quad \sum_{j=1}^{n} x_{j}=\sum_{j=1}^{n} y_{j} .
$$

Also $x$ is said to be weakly-majorized by $y$, denoted by $x \prec_{w} y$, if

$$
\sum_{j=1}^{k} x_{j}^{\downarrow} \leq \sum_{j=1}^{k} y_{j}^{\downarrow} \quad(\text { for } k=1,2, \ldots, n) .
$$

Here $x_{1}^{\downarrow} \geq x_{2}^{\downarrow} \geq \cdots \geq x_{n}^{\downarrow}$ is the non-increasing rearrangement of the components of the vector $x$.

Characterizations of majorization and weak majorization are well studied. Suppose $x, y \in \mathbb{R}^{n}$ with non-negative components; then $x \prec_{w} y$ if and only if $x=D y$ for some doubly sub-stochastic matrix $D$ ([4]). A square matrix $D$ is said to be doubly sub-stochastic if all entries are non-negative and each row sum is at most 1 , and the same for each column sum. Earlier, Littlewood et al, proved that, for $x, y \in \mathbb{R}^{n}, x \prec y$ if and only if $x=D y$ for some doubly stochastic matrix $D$ ([7]). A square matrix $D$ is said to be doubly stochastic if all entries are non-negative and each row sum is equal to 1 , as does each column sum. It is well known that if $x, y \in \mathbb{R}^{n}$ and $x \prec y$, then $x$ is in the convex hull of all vectors obtained by permuting the components of $y([4])$. Multiple studies generalized the notion of majorization using different settings ([8]). In [6], the authors have studied $G$-majorization for a closed group $G \subset M_{n}$ of orthogonal matrices of order $n$. Recently, in $[1,9]$, the authors have introduced the notions of even majorization and $B$-majorization

${ }^{*}$ Received by the editors on February 25, 2020. Accepted for publication on June 8, 2020. Handling Editor: Michael Tsatsomeros. Corresponding Author: G. Sankara Raju Kosuru.

$\dagger$ Department of Mathematics, Indian Institute of Technology Ropar, Rupnagar - 140 001, Punjab, India (raju@iitrpr.ac.in).

${ }^{\ddagger}$ Mathematics Department, Indian Institute of Technology Ropar, Rupnagar - 140 001, Punjab, India (subhajit.saha@iitrpr.ac.in). 
respectively, which are indeed special cases of the aforementioned $G$-majorization, and provide particular geometric set-ups.

Here, we considered a different geometry, a cone type, the convex hull of the origin and vectors obtained by permuting the components of a vector $y$ in $\mathbb{R}^{n}$ and fixing a particular component of $y$. Depending on this, we introduced a new type of majorization, called cone type majorization, which includes weak majorization in the positive cone of $\mathbb{R}^{n}$. We establish a few basic properties and a characterization of this majorization.

One of the interesting problems in the theory of majorization is the linear preserver problem, which analyzes linear operators that preserve majorization on certain spaces. For an overview of such problems the reader can refer to [11]. The following result gives a characterization of linear maps which preserve majorization.

Theorem 1.1. ([2]) Let $T: \mathbb{R}^{n} \rightarrow \mathbb{R}^{n}$ be a linear map. Then $T(x) \prec T(y)$, whenever $x \prec y$ if and only if one the following conditions hold:

(i) $T(x)=\operatorname{tr}(x)$ a for some $a \in \mathbb{R}^{n}$.

(ii) $T(x)=\alpha P(x)+\beta \operatorname{tr}(x)$ e for some $\alpha, \beta \in \mathbb{R}$ and a permutation matrix $P$.

Here $\operatorname{tr}(x)=\sum_{j=1}^{n} x_{j}$ is the trace of the vector $x=\left(x_{1}, x_{2}, \ldots, x_{n}\right)^{t} \in \mathbb{R}^{n}$ and e denotes the vector $(1,1, \ldots, 1)^{t}$ in $\mathbb{R}^{n}$.

In this study, we provide the structure of linear maps on $\mathbb{R}^{n}$, which preserve the cone type majorization. We prove that a linear map $A: \mathbb{R}^{n} \rightarrow \mathbb{R}^{n}$ preserves the cone type majorization if and only if either all the columns of $A$, except the first column, are all equal or the $2^{\text {nd }}$ to the $n^{\text {th }}$ components of the first column of $A$ are all equal and the rest of the columns are possible permutations of the second column, keeping the first component the same. Finally, we studied the structure of such linear maps which strongly preserve the cone type majorization.

2. Cone type majorization. Let $\alpha=\left(\alpha_{1}, \alpha_{2}, \ldots, \alpha_{n}\right)^{t}$ be a vector in $\mathbb{R}^{n}$ and let $\left\{e_{1}, e_{2}, \ldots, e_{n}\right\}$ be the standard orthonormal basis of $\mathbb{R}^{n}$. Suppose $S_{n}$ is the set of all permutations on the $n$ symbols, $\{1,2, \ldots, n\}$. We first fix some notations, which will be used throughout this paper:

$$
\begin{aligned}
\dot{\alpha} & =\left(\alpha_{2}, \ldots, \alpha_{n}\right)^{t} \in \mathbb{R}^{n-1}, \text { for } \alpha=\left(\alpha_{1}, \alpha_{2}, \ldots, \alpha_{n}\right)^{t} \in \mathbb{R}^{n}, \\
\dot{\alpha}^{\downarrow} & =\left(\alpha_{2}^{\downarrow}, \ldots, \alpha_{n}^{\downarrow}\right)^{t}, \text { the non-increasing rearrangement of the components of } \dot{\alpha}, \\
S_{n}^{1} & =\left\{\sigma \in S_{n}: \sigma(1)=1\right\}, \\
\mathscr{M} & =\left\{\Pi: \Pi \text { is a permutation matrix of order } n \text { with } \Pi e_{1}=e_{1}\right\}, \\
S_{x} & =\{\Pi x: \Pi \in \mathscr{M}\} \text { for } x \in \mathbb{R}^{n}, \text { and } \\
e & =(1,1, \ldots, 1)^{t} \in \mathbb{R}^{n} .
\end{aligned}
$$

Definition 2.1. Let $x, y$ be two vectors in $\mathbb{R}^{n}$. We say that $x$ is cone type majorized by $y$ if $x$ belongs to the convex hull of the origin and the points of $S_{y}$. We denote this by $x \prec_{C} y$. Also $x$ is said to be cone type equivalent to $y$, denoted by $x \sim_{C} y$, whenever $x \prec_{C} y$ and $y \prec_{C} x$.

It is evident from the above definition that $\left(\frac{-2}{3}, \frac{4}{3}, \frac{4}{3}\right)^{t} \prec_{C}(-1,1,3)^{t}$.

The following figure shows the (geometric) difference between the cone type majorization and majoriza- 
tion on $\mathbb{R}^{3}$.
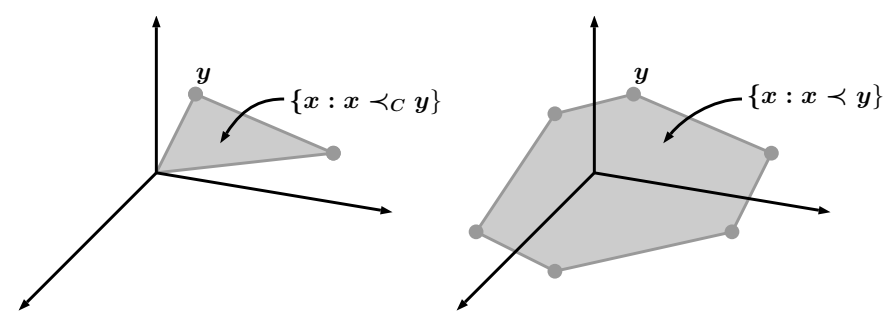

Figure 1. The geometries of the sets $\left\{x: x \prec_{C} y\right\}$ and $\{x: x \prec y\}$ in $\mathbb{R}^{3}$.

We present some basic properties of the cone type majorization. Detailed verifications can be obtained by the reader.

Proposition 2.2. Let $x, y, z$ be any three vectors in $\mathbb{R}^{n}$. Then the following statements hold:

(i) $x \prec_{C} x$.

(ii) If $x \prec_{C} y$ and $y \prec_{C} z$, then $x \prec_{C} z$.

(iii) If $\Pi, \hat{\Pi} \in \mathscr{M}$ and $x \prec_{C} y$, then $\Pi x \prec_{C} \hat{\Pi} y$.

(iv) The set $\left\{z \in \mathbb{R}^{n}: z \prec_{C} x\right\}$ is a polyhedron; in particular, it is convex.

Now, we give a characterization of the cone type majorization.

THEOREM 2.3. Let $x=\left(x_{i}\right)^{t}, y=\left(y_{i}\right)^{t}$ be two vectors in $\mathbb{R}^{n}$. Then $x \prec_{C} y$ if and only if there exists some $r \in[0,1]$ such that

$$
x_{1}=r y_{1}, \quad \sum_{j=2}^{k} x_{j}^{\downarrow} \leq r \sum_{j=2}^{k} y_{j}^{\downarrow} \quad \text { for } \quad 2 \leq k \leq n-1, \quad \text { and } \quad \sum_{j=1}^{n} x_{j}=r \sum_{j=1}^{n} y_{j} .
$$

Proof. Let $x \prec_{C} y$. Then $x=c_{0}(0,0, \ldots, 0)^{t}+c_{1}\left(y_{1}, y_{\sigma_{1}(2)}, y_{\sigma_{1}(3)}, \ldots, y_{\sigma_{1}(n)}\right)^{t}+c_{2}\left(y_{1}, y_{\sigma_{2}(2)}, y_{\sigma_{2}(3)}, \ldots\right.$, $\left.y_{\sigma_{2}(n)}\right)^{t}+\cdots+c_{k}\left(y_{1}, y_{\sigma_{k}(2)}, y_{\sigma_{k}(3)}, \ldots, y_{\sigma_{k}(n)}\right)^{t}$ for some $\sigma_{j} \in S_{n}^{1}, 1 \leq j \leq k$ and $c_{i} \geq 0,0 \leq i \leq k$ with $\sum_{i=0}^{k} c_{i}=1$. Therefore, $x=c_{1} \Pi_{1} y+c_{2} \Pi_{2} y+\cdots+c_{k} \Pi_{k} y$ for some $\Pi_{i} \in \mathscr{M}, 1 \leq i \leq k$. Equating the components, we have $x_{1}=r y_{1}$ and $\dot{x}=M \dot{y}$, where $r=c_{1}+c_{2}+\cdots+c_{k}$ and $M=\left(m_{i j}\right)$ is a matrix of order $n-1$ with non-negative entries with $\sum_{j=1}^{n-1} m_{i j}=r=\sum_{i=1}^{n-1} m_{i j}$, for $1 \leq i, j \leq n-1$. Clearly, $r \in[0,1]$. Now if $r=0$, then (2.1) holds trivially. If $r \neq 0$, then $\dot{x}=$ Drý, where $D=\frac{1}{r} M$. Thus, (2.1) holds.

Conversely, assume that (2.1) holds for some $r \in[0,1]$. If $r=0$, then $x=0$. So $x \prec_{C} y$. Now if $r \neq 0$, then $\dot{x}=c_{1} P_{1} \dot{y}+c_{2} P_{2} \dot{y}+\cdots+c_{k} P_{k} \dot{y}$, where $P_{i}$ is a permutation matrix and $c_{j} \geq 0$ for $1 \leq i, j \leq k$ with $\sum_{j=1}^{k} c_{j}=r$. Therefore,

$$
\dot{x}=c_{1}\left(y_{\sigma_{1}(2)}, y_{\sigma_{1}(3)}, \ldots, y_{\sigma_{1}(n)}\right)^{t}+\cdots+c_{k}\left(y_{\sigma_{k}(2)}, y_{\sigma_{k}(3)}, \ldots, y_{\sigma_{k}(n)}\right)^{t}
$$

for some $\sigma_{i} \in S_{n}^{1}, \quad 1 \leq i \leq k$. Hence, $x \prec_{C} y$. 
Let $x=\left(x_{i}\right)^{t}, y=\left(y_{i}\right)^{t}$ be two non-zero vectors in $\mathbb{R}^{n}$ with $x \sim_{C} y$. Then by Theorem 2.3 , there exist $r, r_{1} \in[0,1]$ such that $x_{1}=r y_{1}$ and $\dot{x} \prec r \dot{y}$; also $y_{1}=r_{1} x_{1}$ and $\dot{y} \prec r_{1} \dot{x}$. If $x_{1} \neq 0$, then $r=r_{1}=1$. Therefore, $\dot{x} \prec \dot{y}$ and $\dot{y} \prec x$. Hence, $\dot{x}=P \dot{y}$, where $P$ is a permutation matrix of order $n-1$. On the other hand, if $x_{1}=0$, then $\dot{x} \neq 0$. Thus, we have $r=r_{1}=1$. Therefore, $\dot{x} \prec \dot{y}$ and $\dot{y} \prec \dot{x}$. Thus, $\dot{x}=Q \dot{y}$, where $Q$ is a permutation matrix of order $n-1$. Hence, we have the following lemma.

Lemma 2.4. Let $x, y$ be two vectors in $\mathbb{R}^{n}$. If $x \sim_{C} y$, then $x=\Pi y$ for some $\Pi \in \mathscr{M}$.

Also, it is to be observed that if $r=1$ in (2.1), then the cone type majorization coincides with majorization.

ExAmple 2.5. Let $x=\left(\frac{2}{3}, \frac{4}{3}, \frac{4}{3}\right)^{t}, y=(1,1,3)^{t}, u=(0,1,1)^{t}$ and $v=(1,1,0)^{t}$. Then $x \prec_{C} y$ but $x$ is not majorized by $y$. On the other hand, $u \prec v$ but $u$ is not cone type majorized by $v$.

Let $x, y \in \mathbb{R}^{n}$ be two vectors in $\mathbb{R}^{n}$. Suppose $x \prec_{C} y$. Then by Theorem 2.3, we have $x=K y$, where $K=\left(k_{i j}\right)$ is a matrix of order $n$ with non-negative entries, $k_{11}=r, k_{1 t}=0=k_{t 1}$, for $2 \leq t \leq n$ and $\sum_{j=2}^{n} k_{i j}=r=\sum_{i=2}^{n} k_{i j}$ for $2 \leq i, j \leq n$. Since $r \in[0,1], K$ is doubly sub-stochastic. Thus, the following holds:

FACT 2.6. Let $x$ and $y$ be two vectors in $\mathbb{R}^{n}$ with non-negative components. If $x \prec_{C} y$, then we have $x \prec_{w} y$.

The following example illustrates that the converse of the above fact is not true in general.

ExAmple 2.7. Let $x=\left(\frac{5}{6}, \frac{2}{3}, \frac{1}{3}\right)^{t}$ and $y=(1,1,1)^{t}$. It is easy to see that $x \prec_{w} y$. But $x$ is not cone type majorized by $y$.

Also, it is important to note that, if $x=(0,-1,-1)^{t}$ and $y=(0,-2,-2)^{t}$, then $x \prec_{C} y$ but $x$ is not weakly majorized by $y$. Hence, the non-negativity of the components of $x$ and $y$ is necessary in the Fact 2.6.

3. Linear preservers of the cone type majorization. In this section, we characterize all the linear maps which preserve the cone type majorization.

Definition 3.1. A linear map $A: \mathbb{R}^{n} \rightarrow \mathbb{R}^{n}$ is said to be a preserver of the cone type majorization, if $A x \prec_{C} A y$ whenever $x \prec_{C} y$, for $x, y \in \mathbb{R}^{n}$. We denote the set of all such linear maps by $\mathscr{P}_{p r}\left(\mathbb{R}^{n}\right)$.

It is easy to observe that for $\alpha \in \mathbb{R}$ and $A, B \in \mathscr{P}_{p r}\left(\mathbb{R}^{n}\right), \alpha A, A B \in \mathscr{P}_{p r}\left(\mathbb{R}^{n}\right)$. Also it is clear from Proposition 2.2 (iii) that $\mathscr{M} \subseteq \mathscr{P}_{p r}\left(\mathbb{R}^{n}\right)$. The following example ensures that the inclusion is proper.

Example 3.2. Let $0 \neq \beta \in \mathbb{R}$ be fixed. Suppose $A: \mathbb{R}^{n} \rightarrow \mathbb{R}^{n}$ is the map defined by $A x=\beta \cdot \operatorname{tr}(x) e$ for $x=\left(x_{i}\right)^{t} \in \mathbb{R}^{n}$. Clearly, $A$ is a linear map on $\mathbb{R}^{n}$. Now for $x=\left(x_{i}\right)^{t}, y=\left(y_{i}\right)^{t} \in \mathbb{R}^{n}$, if $x \prec_{C} y$, then by Theorem 2.3, $\operatorname{tr}(x)=r \operatorname{tr}(y)$. Hence, $A$ preserves the cone type majorization.

Now we provide a family of linear maps which preserve the cone type majorization.

Theorem 3.3. Let $A$ be a linear map on $\mathbb{R}^{n}$. Suppose $A$ is of one of the following forms:

(i) $A x=x_{1} a+\operatorname{tr}(\dot{x}) b$, where $a, b \in \mathbb{R}^{n}$ and $x=\left(x_{1}, x_{2}, \ldots, x_{n}\right)^{t}$. 
(ii)

$$
A=\left(\begin{array}{cc}
\alpha & \beta e^{t} \\
\gamma \dot{e} & \lambda P+\delta J
\end{array}\right),
$$

where $\alpha, \beta, \gamma, \lambda, \delta \in \mathbb{R}$ with $\lambda \neq 0, P$ is a permutation matrix of order $n-1$ and $J$ is the matrix of order $n-1$ with all entries 1 .

Then A preserves the cone type majorization.

Proof. Let $x \prec_{C} y$. Then there exists $r \in[0,1]$ such that $x_{1}=r y_{1}$ and $x \prec r y$. If $(i)$ holds, then $A x=r A y$, and hence, $A x \prec_{C} A y$. If $(i i)$ holds, then

$$
A x=\left(\begin{array}{cc}
\alpha & \beta e^{t} \\
\gamma \dot{e} & \lambda P+\delta J
\end{array}\right)\left(\begin{array}{c}
x_{1} \\
\dot{x}
\end{array}\right)=\left(\begin{array}{c}
\alpha x_{1}+\beta \operatorname{tr}(\dot{x}) \\
\gamma x_{1} \dot{e}+(\lambda P+\delta J) \dot{x}
\end{array}\right)=\left(\begin{array}{c}
r\left(\alpha y_{1}+\beta \operatorname{tr}(\dot{y})\right) \\
\gamma x_{1} \dot{e}+(\lambda P+\delta J) \dot{x}
\end{array}\right) .
$$

Since $\dot{x} \prec r \dot{y},(\lambda P+\delta J) \dot{x} \prec r(\lambda P+\delta J) \dot{y}$, and hence, $\gamma x_{1} \dot{e}+(\lambda P+\delta J) \dot{x} \prec r\left(\gamma y_{1} \dot{e}+(\lambda P+\delta J) \dot{y}\right)$. This implies that $A x \prec_{C} A y$. Hence, $A$ preserves the cone type majorization.

The converse of the above theorem is true. The following lemma will be useful in this direction.

LEMmA 3.4. Let $A: \mathbb{R}^{n} \rightarrow \mathbb{R}^{n}$ be a linear map. Then the following are equivalent:

(i) A preserves the cone type majorization.

(ii) For any $\Pi \in \mathscr{M}$, there exists $\hat{\Pi} \in \mathscr{M}$ such that $A \Pi=\hat{\Pi} A$.

Proof. Let $A$ be a preserver of the cone type majorization. Now

$$
A x=\left(\left\langle x, a_{1}^{t}\right\rangle,\left\langle x, a_{2}^{t}\right\rangle, \ldots,\left\langle x, a_{n}^{t}\right\rangle\right)^{t},
$$

where $a_{i}$ denotes the $i^{\text {th }}$ row of the matrix of $A$ with respect to the standard basis. It is enough to prove that for any $\Pi \in \mathscr{M}$, there exists a permutation $\sigma \in S_{n}^{1}$ such that

$$
a_{1} \Pi=a_{1} \quad \text { and } \quad a_{j} \Pi=a_{\sigma(j)} \text { for } j=2,3, \ldots, n \text {. }
$$

Let $\Pi \in \mathscr{M}$. By Lemma 2.4 and the fact that $\Pi x \sim_{C} x$, we have $A \Pi x=\Pi_{1} A x$ for some $\Pi_{1} \in \mathscr{M}$. Now as $A x=\left(\left\langle x, a_{1}^{t}\right\rangle,\left\langle x, a_{2}^{t}\right\rangle, \ldots,\left\langle x, a_{n}^{t}\right\rangle\right)^{t}$, we have

$$
A \Pi x=\left(\left\langle x, \Pi^{t} a_{1}^{t}\right\rangle,\left\langle x, \Pi^{t} a_{2}^{t}\right\rangle, \ldots,\left\langle x, \Pi^{t} a_{n}^{t}\right\rangle\right)^{t}
$$

where $\Pi^{t}$ is the transpose of $\Pi$. By equating the components of $\Pi_{1} A x$ and $A \Pi x$ we get $\left\langle x, a_{1}^{t}\right\rangle=\left\langle x, \Pi^{t} a_{1}^{t}\right\rangle$ and $\left\langle x, a_{\sigma(j)}^{t}\right\rangle=\left\langle x, \Pi^{t} a_{j}^{t}\right\rangle$ for some $\sigma \in S_{n}^{1}, 2 \leq j \leq n$. This implies (3.3).

Conversely, assume that $x \prec_{C} y$. Then $x=c_{0} 0+c_{1} \Pi_{1} y+c_{2} \Pi_{2} y+\cdots+c_{k} \Pi_{k} y$, where 0 is the origin, $\Pi_{i} \in \mathscr{M}$ for $1 \leq i \leq k$, and $c_{j} \geq 0$ for $0 \leq j \leq k$ with $\sum_{j=0}^{k} c_{j}=1$. Therefore, we have $A x=$ $c_{0} 0+c_{1} \hat{\Pi}_{1} A y+c_{2} \hat{\Pi}_{2} A y+\cdots+c_{k} \hat{\Pi}_{k} A y$, where $\hat{\Pi}_{i} \in \mathscr{M}$ for $1 \leq i \leq k$. Thus, $A x \prec C A y$. This completes the proof.

Now we state an elementary fact, which will be useful in the sequel. The proof of the fact easily follows from combinatorial techniques. 
FACT 3.5. Let $a=\left(a_{1}, a_{2}, a_{3}, \ldots, a_{n}\right)^{t}$ be a vector in $\mathbb{R}^{n}$. All the components of á are equal except one, if and only if $\left\{a^{t} \Pi: \Pi \in \mathscr{M}\right\}$ contains exactly $n-1$ elements.

Now we prove the main theorem in this section.

THEOREM 3.6. Let $A$ be a linear map on $\mathbb{R}^{n}$. Then the following are equivalent:

(a) A preserves the cone type majorization.

(b) $A$ is of one of the following forms:

(i) $A x=x_{1} a+\operatorname{tr}(x) b$, where $a, b \in \mathbb{R}^{n}$ and $x=\left(x_{1}, x_{2}, \ldots, x_{n}\right)^{t}$.

(ii)

$$
A=\left(\begin{array}{cc}
\alpha & \beta e^{t} \\
\gamma e ́ & \lambda P+\delta J
\end{array}\right)
$$

where $\alpha, \beta, \gamma, \lambda, \delta \in \mathbb{R}$ with $\lambda \neq 0, P$ is a permutation matrix of order $n-1$ and $J$ is the matrix of order $n-1$ with all entries 1 .

Proof. Let $A$ preserve the cone type majorization and let the matrix of $A$ be represented in the form $\left[a_{1} / a_{2} / \ldots / a_{n}\right]$, with respect to the standard basis, where $a_{i}$ denotes the $i^{t h}$ row of the matrix of $A$. By (3.3) we have that for any $\Pi \in \mathscr{M}$, there exists a permutation $\sigma \in S_{n}^{1}$ such that

$$
a_{1} \Pi=a_{1} \quad \text { and } \quad a_{j} \Pi=a_{\sigma(j)} \text { for } 2 \leq j \leq n
$$

As $a_{1} \Pi=a_{1}$ for any $\Pi \in \mathscr{M}$, the $2^{\text {nd }}$ to $n^{\text {th }}$ components of $a_{1}$ are all equal. Now if all but one component of $a_{2}^{\prime}$ (or any $\left.a_{k}^{\prime}, 2 \leq k \leq n\right)$ are the same, then by Fact 3.5 , the orbit $\left\{a_{2} \Pi: \Pi \in \mathscr{M}\right\}$ contains exactly $n-1$ distinct elements. Thus, by (3.3), the orbit $\left\{a_{2} \Pi: \Pi \in \mathscr{M}\right\}$ coincides with $\left\{a_{2}, a_{3}, \ldots, a_{n}\right\}$. Therefore, $A$ is of the form $(i i)$. Now if $a_{2}^{\prime}$ (or any $a_{k}^{\prime}, 2 \leq k \leq n$ ) is a scalar multiple of $e^{t}$, it is easy to observe that $A$ is of the form $(i)$. No other case is possible, otherwise the orbit $\left\{a_{2} \Pi: \Pi \in \mathscr{M}\right\}$ would contain more than $n-1$ distinct elements, which contradicts (3.3). The converse is contained in Theorem 3.3. This completes the proof.

The following example shows that the sum of two cone type majorization preservers on $\mathbb{R}^{n}$ need not be a cone type majorization preserver.

ExAmple 3.7. Let $A=\left[\begin{array}{llll}1 & 3 & 3 & 3 \\ 4 & 1 & 2 & 2 \\ 4 & 2 & 1 & 2 \\ 4 & 2 & 2 & 1\end{array}\right]$ and $B=\left[\begin{array}{llll}1 & 1 & 1 & 1 \\ 4 & 3 & 3 & 1 \\ 4 & 3 & 1 & 3 \\ 4 & 1 & 3 & 3\end{array}\right]$. Then by Theorem 3.6, the maps $A$ and $B$ both are in $\mathscr{P}_{p r}\left(\mathbb{R}^{4}\right)$. Let $M=A+B=\left[\begin{array}{llll}2 & 4 & 4 & 4 \\ 8 & 4 & 5 & 3 \\ 8 & 5 & 2 & 5 \\ 8 & 3 & 5 & 4\end{array}\right]$. By Theorem 3.6, $M$ is not a preserver of the cone type majorization.

For $n=3$, a simple calculation gives us that $A+B \in \mathscr{P}_{p r}\left(\mathbb{R}^{3}\right)$, if $A, B \in \mathscr{P}_{p r}\left(\mathbb{R}^{3}\right)$ and $A, B$ both are of the same form i.e. both are either of the form $(i)$ or of the from $(i i)$. 
4. Strong preservers of the cone type majorization. In this section, we study the structure of linear maps which strongly preserve the cone type majorization.

Definition 4.1. A linear map $A: \mathbb{R}^{n} \rightarrow \mathbb{R}^{n}$ is said to be a strong preserver of the cone type majorization if the following holds true:

$$
x \prec_{C} y \text { if and only if } A x \prec_{C} A y, \quad \text { for } x, y \in \mathbb{R}^{n} \text {. }
$$

We denote the set of all such maps by $\mathscr{P}_{p r}^{s}\left(\mathbb{R}^{n}\right)$.

It is evident from the above definition that for $\alpha(\neq 0) \in \mathbb{R}$ and $A, B \in \mathscr{P}_{p r}^{s}\left(\mathbb{R}^{n}\right), \alpha A, A B \in \mathscr{P}_{p r}^{s}\left(\mathbb{R}^{n}\right)$. The following example shows that a cone type majorization preserver on $\mathbb{R}^{n}$ is not necessarily a strong preserver of the cone type majorization.

ExAmple 4.2. Let $e$ be the vector $(1,1,1)^{t}$ and $A: \mathbb{R}^{3} \rightarrow \mathbb{R}^{3}$ be the linear map defined by $A x=\operatorname{tr}(x) . e$, for $x \in \mathbb{R}^{3}$. Then by Theorem 3.6, $A \in \mathscr{P}_{p r}\left(\mathbb{R}^{3}\right)$. But $A \notin \mathscr{P}_{p r}^{s}\left(\mathbb{R}^{3}\right)$, since $A(1,-1,0)^{t}$ is cone type majorized by $A(0,0,0)^{t}$, but $(1,-1,0)^{t}$ is not cone type majorized by $(0,0,0)^{t}$.

Let $A$ be a linear map on $\mathbb{R}^{n}$. Suppose $A x=0$, for some $x \in \mathbb{R}^{3}$. Then $A x$ is cone type majorized by $0=A 0$. If $A$ strongly preserves the cone type majorization, then $x \prec_{C} 0$. Hence, $x=0$. Thus, we have:

THEOREM 4.3. Let $A$ be a linear map on $\mathbb{R}^{n}$ that strongly preserves the cone type majorization. Then $A$ is invertible.

Lemma 4.4. Let $A$ be an invertible linear map on $\mathbb{R}^{n}$. Then $A$ preserves the cone type majorization if and only if $A^{t}$ preserves the cone type majorization, where $A^{t}$ denotes the transpose of $A$.

Proof. Let $A$ be a preserver of the cone type majorization. As $A$ is invertible, $A$ is of the form ( $i i)$ of the Theorem 3.6. Clearly, $A^{t}$ is also of the same form. Therefore, $A^{t}$ is also a preserver of the cone type majorization. In a similar fashion, one can establish the converse.

The next example shows that the invertibility condition of the linear map $A$, in the above lemma is necessary.

Example 4.5. Let $A=\left[\begin{array}{lll}1 & 2 & 2 \\ 3 & 4 & 4 \\ 5 & 6 & 6\end{array}\right]$. Clearly, $A$ is not invertible and $A^{t}=\left[\begin{array}{lll}1 & 3 & 5 \\ 2 & 4 & 6 \\ 2 & 4 & 6\end{array}\right]$. Then $A^{t}$ is not a preserver of the cone type majorization.

Theorem 4.6. Suppose $A$ is an invertible linear map on $\mathbb{R}^{n}$. Then $A$ is a preserver of the cone type majorization if and only if $A^{-1}$ is a preserver of the cone type majorization.

Proof. Assume that $A$ is a preserver of the cone type majorization. Let $\Pi \in \mathscr{M}$. Then there exists a $\hat{\Pi} \in \mathscr{M}$ such that $A \Pi^{t}=\hat{\Pi} A$. Therefore, $\Pi^{t} A^{-1}=A^{-1} \hat{\Pi}$, which implies $\left(A^{-1}\right)^{t} \Pi=\hat{\Pi}^{t}\left(A^{-1}\right)^{t}$. By Lemma 3.4, $\left(A^{-1}\right)^{t}$ is a preserver of the cone type majorization. Hence, by Lemma $4.4, A^{-1}$ is a preserver of the cone type majorization. In a similar fashion, one can prove the converse.

As a consequence of Theorem 3.6, Theorem 4.3 and Theorem 4.6 we get the following:

THEOREM 4.7. Let $A$ be a linear map on $\mathbb{R}^{n}$. Then the following are equivalent:

(i) A is a strong preserver of the cone type majorization.

(ii) $A$ is invertible and $A$ preserves the cone type majorization. 
(iii) $A$ is invertible and $A$ is of the form:

$$
A=\left(\begin{array}{cc}
\alpha & \beta e^{t} \\
\gamma e^{\prime} & \lambda P+\delta J
\end{array}\right),
$$

where $\alpha, \beta, \gamma, \lambda, \delta \in \mathbb{R}$ with $\lambda \neq 0, P$ is a permutation matrix of order $n-1$ and $J$ is the matrix of order $n-1$ with all entries 1 .

Let $A$ be a linear map on $\mathbb{R}^{n}$. If $A$ preserves the cone type majorization, then in view of Theorem 3.3, $A$ is not necessarily invertible. Suppose $A$ is of the form

$$
A=\left(\begin{array}{cc}
\alpha & \beta e^{t} \\
\gamma \dot{e} & \lambda P+\delta J
\end{array}\right),
$$

with $(\alpha, \beta) \neq(0,0),(\alpha, \gamma) \neq(0,0), \lambda \neq 0$ and

$$
\begin{cases}\lambda \neq-(n-1) \delta & \text { if } \beta=0 \\ (\alpha, \gamma) \neq((n-1) c \beta, c(\lambda+(n-1) \delta)), \text { where } c \in \mathbb{R} \text { with } c \neq 0 & \text { if } \beta \neq 0\end{cases}
$$

Then $A$ is invertible.

Acknowledgements. The authors gratefully thank Dr. Srivatsava Naidu (Indian Institute of Technology Ropar) and the reviewers for their comments and constructive suggestions for the betterment of the manuscript.

\section{REFERENCES}

[1] T. Ando. Linear preservers of even majorization on $M_{n, m}$. Linear and Multilinear Algebra, 62(11):1437-1449, 2014.

[2] T. Ando. Majorization, doubly stochastic matrices, and comparison of eigenvalues. Linear Algebra and its Applications, 118:163-248, 1989.

[3] W. Arveson and R.V. Kadison. Diagonals of self-adjoint operators. Contemporary Mathematics, Operator Theory, Operator Algebras, and Applications, Amer. Math. Soc., Providence, RI, 414:247-263, 2006.

[4] R. Bhatia. Matrix Analysis. Springer-Verlag, New York, 1997.

[5] N. Canosa, R. Rossignoli, and M. Portesi. Majorization relations and disorder in generalized statistics. Physica A: Statistical Mechanics and its Applications, 371(1):126-129, 2006.

[6] M.L. Eaton. On group induced orderings, monotone functions, and convolution theorems. Inequalities in Statistics and Probability (Lincoln, Neb., 1982). 5:1325, IMS Lecture Notes Monographs, Inst. Math. Statist., Hayward, CA, 1984.

[7] G.H. Hardy, J.E. Littlewood, and G. Pólya. Inequalities, second edition. Cambridge University Press, Cambridge, 1952.

[8] A.W. Marshall, I. Olkin, and B.C. Arnold. Inequalities: Theory of Majorization and its Applications, second edition. Springer, New York, 2011.

[9] M.A.H. Nadoshan and A. Armandnejad. B-majorization and its linear preservers. Linear Algebra and its Applications, 478:218-227, 2015.

[10] M.A. Nielsen and G. Vidal. Majorization and the interconversion of bipartite states. Quantum Information $\mathcal{E}$ Computation, 1(1):76-93, 2001.

[11] S. Pierce, M.H. Lim, R. Loewy, C.-K. Li, N.K. Tsing, B McDonald, and L. Beasley. A survey of linear preserver problems. Linear and Multilinear Algebra, 33:1-129, 1992. 